\title{
Nanostructured Electrodes for Improved Neural Recording
}

\author{
Karen C. Cheung', Yang-Kyu Choi ${ }^{2}$, Tim Kubow' ${ }^{1}$, and Luke P. Lee ${ }^{1}$
}

(1) Berkeley Sensor \& Actuator Center and Department of Bioengineering, University of California, Berkeley

(2) Department of Electrical Engineering and Computer Science, University of California, Berkeley

\begin{abstract}
We present a new method of increasing the effective electrode surface for improved neural recording. To optimize the electrode, the impedance can be decreased by introducing surface roughness or nanostructures on the electrode. High aspect ratio pillar-like polysilicon nanostructures are created in a reactive ion etch. Nanostructure robustness in cell culture is examined.
\end{abstract}

\section{INTRODUCTION}

The interface between neurons and electrodes is one of the key issues in implantable microdevices and bioelectronics. The ideal electrode requires maximum selectivity and minimum impedance. During in vivo experiments, electrode insertion inevitably results in tissue damage, and signals from living cells must travel through the attenuating layer of damaged cells. Selectivity refers to the ability to select a single neuron from a multitude of interconnected cells or even damaged cells. High impedance in an electrode attenuates and filters the measured signal. In addition, a sufficient signal-to-noise ratio is required for data analysis; low electrode impedance gives high signal gain [1]. However, an increase in electrode selectivity with smaller geometric electrode size results in increased impedance and noise. To optimize the electrode, the impedance per unit of geometric surface area must be decreased by altering its nanostructure. With the introduction of surface roughness or nanostructures, a square $20 \mu \mathrm{m} \times 20 \mu \mathrm{m}$ electrode has only 400 square microns of geometric (planar) area but a much larger effective area.

Effective electrode surface area can be increased through micropatterning, surface roughening, and chemical modification [2]. In micropatterning, structures such as grooves or holes are fabricated on the electrodes. If this pattern is etched into the substrate, the metal that forms the electrode must then be deposited conformally. Although an aspect ratio as high as 100:1 can be achieved using deep reactive ion etching, the metal deposition method (e.g. evaporation or sputtering) ultimately limits the aspect ratio and thus the final effective surface area of the micropatterned structures. These structures are typically on the micro scale. Another method of fabricating such structures is by first photolithographically defining the structures on top of the electrode and then electroplating metal using photoresist as a mold.

Electrode materials historically used in neuroscience are gold, platinum, and iridium. The most common surface modifications are wet chemical etching for gold, electroplating platinum black or ion milling the platinum surface, or activating iridium to form iridium oxide. However, they suffer from some drawbacks such as harsh chemical treatment in the case of gold etching, and poor adhesion of platinum powder to the electrode $[3,4]$. 
The junction between the cell and the electrode can be described by equivalent circuits which have the following elements: the space charge layer, charge transfer resistance, Warburg impedances, spreading resistance, shunt resistance, and other parasitic capacitances. Because the total electrode impedance is approximately proportional to the effective surface area, a 10 times increase in surface area should give an order of magnitude decrease in impedance.

We present a new method of increasing the effective electrode surface for improved neural recording. The introduction of nanostructures on the electrode pads will significantly increase the effective electrode surface area and decrease impedance. High aspect ratio pillar-like polysilicon nanostructures are created in a reactive ion etch. Nanostructure robustness in cell culture is examined.

increased effective electrode surface area

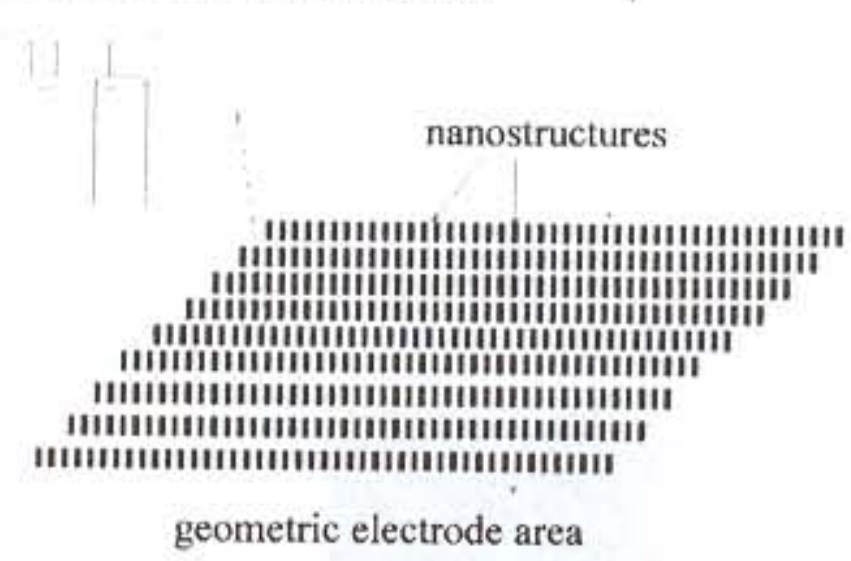

geometric electrode area

Fig. I(a) Nanostructures on an electrode increase the effective surface area.

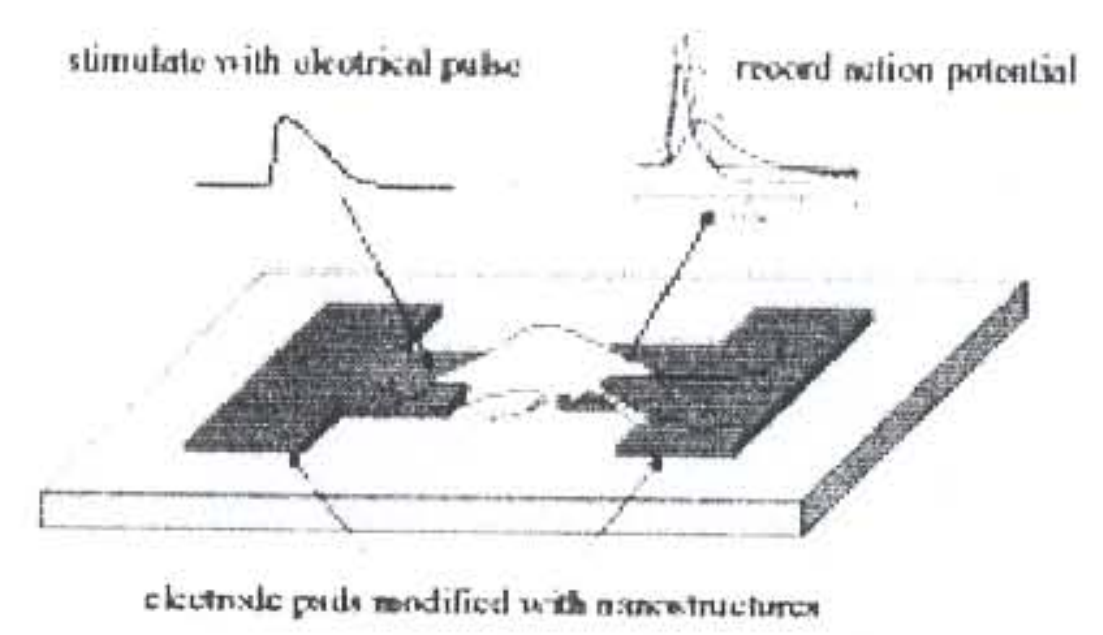

Fig. I(b) Schematic of experimental setup for nanostructured electrodes.

\section{MATERIALS AND METHODS}

\section{Nanostructure Fabrication:}

The substrate was a $1 \mu \mathrm{m}$ film of heavily n-type in-situ doped polysilicon, which was deposited by low-pressure chemical vapor deposition (LPCVD) onto an oxide-coated silicon wafer. It was annealed at $900^{\circ} \mathrm{C}$ for 30 minutes to activate the dopants in the polysilicon.

1. Electrode pads were defined by conventional I-line lithography and a two-step anisotropic plasma etch (Lam Research 9400 TCP): a breakthrough step removes the native oxide and an etch step patterns the polysilicon.

2. After stripping the photoresist, nano-pillars are formed on the electrodes, also by using a two-step anisotropic plasma etch.

The Lam Research 9400 TCP etcher is a single wafer, plasma reactive ion etch (RIE) system that has top and bottom powered parallel electrode plates. $\mathrm{CF}_{4}$ is used during the initiation phase of the etch (breakthrough step) to remove the native oxide. $\mathrm{Cl}_{2}$ is the main etch gas for polysilicon. $\mathrm{HBr}$ is used to improve the selectivity between polysilicon and oxide; $\mathrm{O}_{2}$ is used in conjunction with $\mathrm{HBr}$ because it passivates the polysilicon during etching. 
Table 1. Etch Parameters

\begin{tabular}{|c|c|c|c|}
\hline Structure: & Breakthrough Step & Etch step & Polysilicon Etch rate \\
\hline 1. Electrodes & 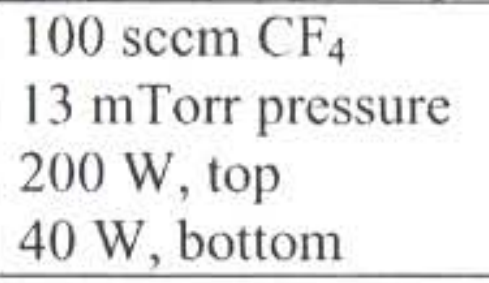 & $\begin{array}{l}50 \mathrm{sccm} \mathrm{Cl}_{2}+150 \mathrm{sccm} \mathrm{HBr} \\
15 \mathrm{mTorr} \text { pressure } \\
300 \mathrm{~W} \text {, top } \\
150 \mathrm{~W} \text {, bottom }\end{array}$ & $\begin{array}{l}550 \mathrm{~nm} / \mathrm{min} \\
20: 1 \text { selectivity } \\
\text { (polysilicon:oxide) }\end{array}$ \\
\hline 2. Nano-pillars & $\begin{array}{l}\text { (short } 10 \text { sec etch) } \\
100{\text { sccm } \mathrm{CF}_{4}} \\
13 \mathrm{~m} \text { Torr pressure } \\
200 \mathrm{~W} \text {, top } \\
40 \mathrm{~W} \text {, bottom }\end{array}$ & $\begin{array}{l}200 \mathrm{sccm} \mathrm{HBr}+5 \mathrm{sccm} \mathrm{O}_{2} \\
35 \mathrm{mT} \text { orr pressure } \\
250 \mathrm{~W} \text {, top } \\
120 \mathrm{~W} \text {, bottom }\end{array}$ & $\begin{array}{l}480 \mathrm{~nm} / \mathrm{min} \\
200: 1 \text { selectivity } \\
\text { (polysilicon:oxide) }\end{array}$ \\
\hline
\end{tabular}

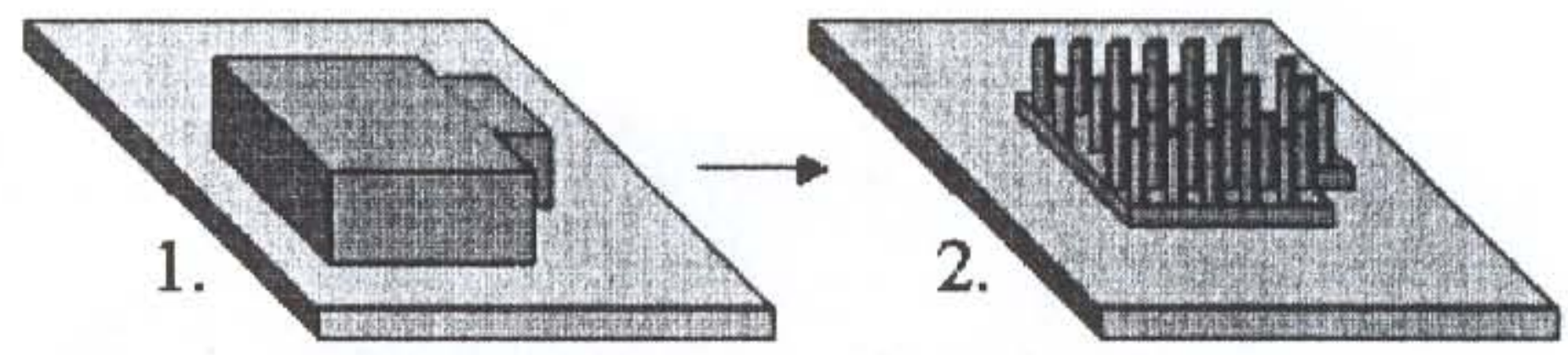

Fig. 2. Nanostructure fabrication:

1. A polysilicon film is deposited on an oxide surface and patterned into the electrode shape;

2. The film is etched back in a highly selective reactive ion etch, which produces the nanostructures.

\section{Neuron Dissociation and Culture:}

Embryos were rapidly removed on embryonic day 18 following cervical dislocation of a pregnant rat. The embryos were decapitated, and the brains were carefully removed and placed in warm HEPES-buffered saline solution. The hippocampi were removed and treated with trypsin for $15 \mathrm{~min}$ at $37^{\circ} \mathrm{C}$, followed by washing and gentle trituration. The dissociated cells were plated at densities of $20,000-50,000 \mathrm{cells} / \mathrm{ml}$ on poly-L-lysine treated nanostructurepatterned substrates in $35 \mathrm{~mm}$ Petri dishes. The plating medium was DMEM supplemented with $10 \%$ heat-inactivated fetal bovine serum, $10 \%$ Ham's F12 with glutamine and $50 \mathrm{U} / \mathrm{ml}$ penicillin-streptomycin. Twenty-four hours after plating, the culture medium was changed to the above medium containing $20 \mathrm{mM} \mathrm{KCl}$. Medium was partially exchanged 3 times each week. Cultures prepared in this manner provide neurons that can survive in vitro for 8 weeks.

\section{Biological Sample Preparation for SEM:}

The cultured samples were fixed in $2 \%$ glutaraldehyde in $0.1 \mathrm{M}$ sodium cacodylate buffer, $\mathrm{pH}$ 7.2. After a post-fix in $1 \%$ osmium tetroxide in $0.1 \mathrm{M}$ sodium cacodylate buffer and rinsing, 
the samples were dehydrated in ethanol and dried in the critical point dryer. The samples were mounted onto stubs using carbon tape and coated with $5 \mathrm{~nm}$ carbon by evaporation.

\section{RESULTS AND DISCUSSIONS}

The nanostructures were densely packed as shown in Fig. 3(a). The pillars were $0.3 \mu \mathrm{m}$ tall, nd their diameters ranged from $20 \mathrm{~nm}$ to $100 \mathrm{~nm}$. This gives a greater than 20 times increase in the surface area of the substrate. Fig. 3(b) shows the nanostructures on top of each electrode pad increase the effective electrode surface area and hopefully decrease impedance during cell recording.

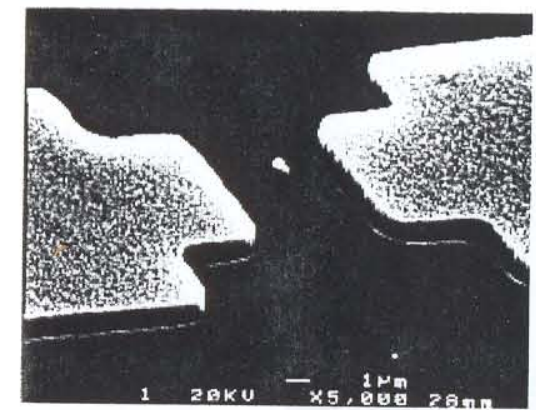

Fig. 3 (a) The nanostructures on top of each electrode pad.

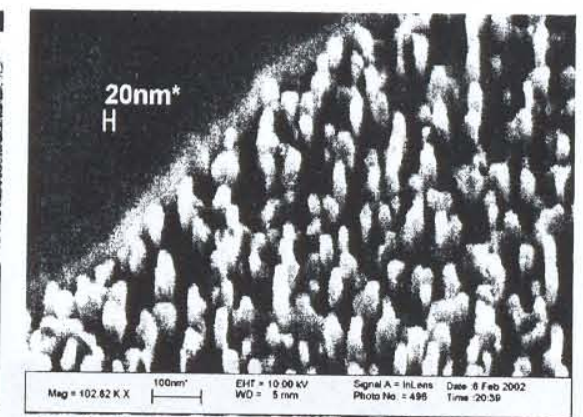

Fig. 3 (b) An SEM image of the pillars. The pillars are $20 \mathrm{~nm}$ in diameter.

A change in the etch parameters explains the different etch selectivity (compare the electrode and nano-pillar etch steps in Table 1) and why both microscale electrode pads and nanoscale pillars can be made in the same etcher. During the nano-pillar etch step, native oxide and dust on the surface of the polysilicon serve as micromasks. This mask initiates the formation of nanopillars in the highly directional etch. As the nano-pillars are etched down further, they are passivated with a thin layer of silicon oxybromide because $\mathrm{O}_{2}$ acts as oxidant to generate $\mathrm{Si}_{\mathrm{x}} \mathrm{O}_{\mathrm{y}} \mathrm{Br}_{\mathrm{z}}$. This continuous passivation prevents the $\mathrm{HBr}$ from etching the silicon underneath resulting in taller and taller nano-pillars with continued etching. The density and diameter of the nano-pillars are controlled by the ratio of $\mathrm{HBr}$ and $\mathrm{O}_{2}$.

After two weeks in culture, it was obvious that our preparations were infected with bacteria; only a few hippocampus neurons remained in the culture. SEMs show that the bacteria strongly prefer to attach to the nanostructure-patterned areas.
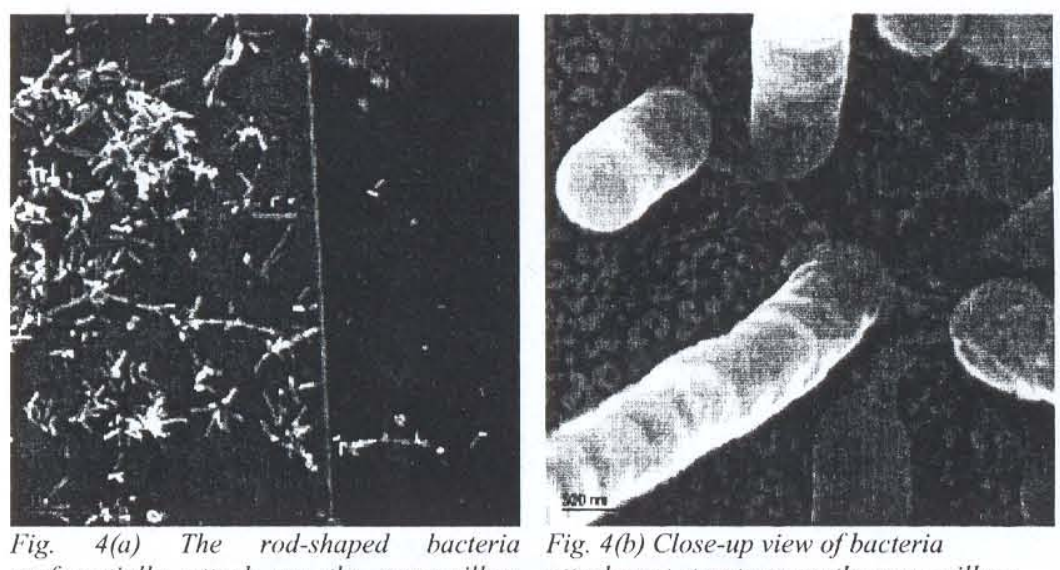

preferentially attach on the nano-pillars attachment structures on the nano-pillars: (left) and not on the unpatterned surface (right)

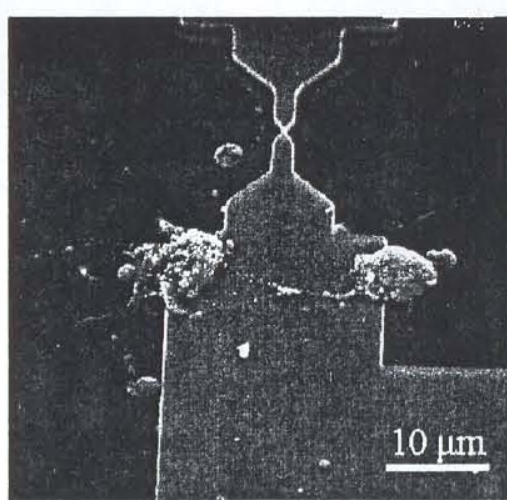

Fig. 5(a) Two neurons on an electrode pad.

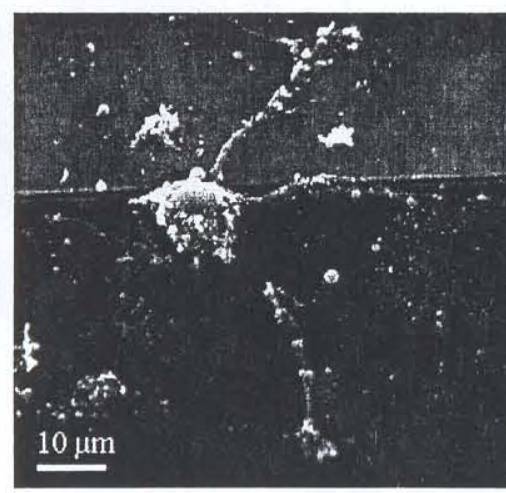

Fig. 5(b) A neuron extending a process onto a nano-pillar region (top).
Neuronal response to electrical stimulation will be recorded from electrodes that have nanostructures and compared to recordings from plain electrodes to study electrode impedance as shown in Fig. 1(b) 


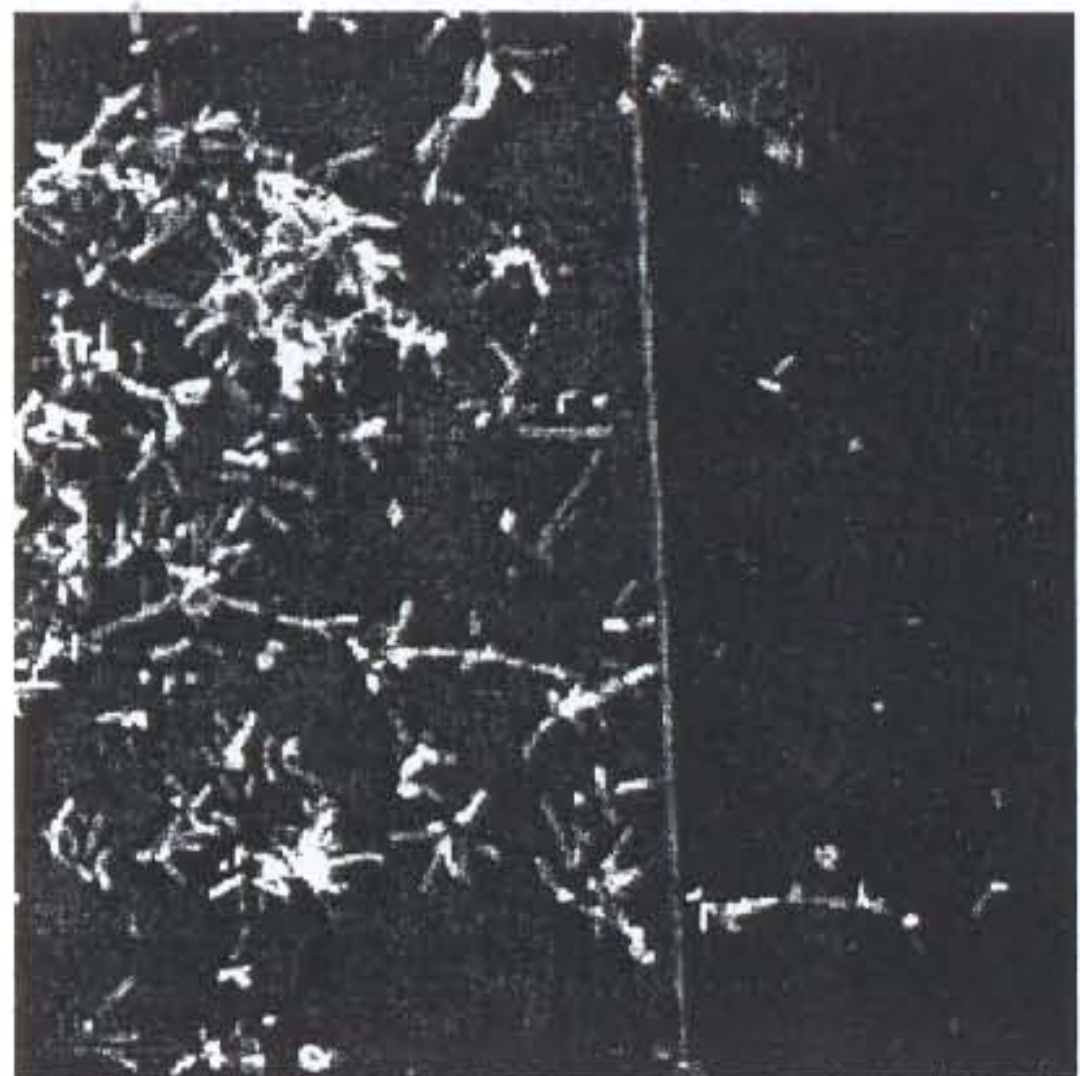

Fig. 4(a) The rod-shaped bacteria preferentially attach on the nano-pillars (left) and not on the unpatterned surface (right).

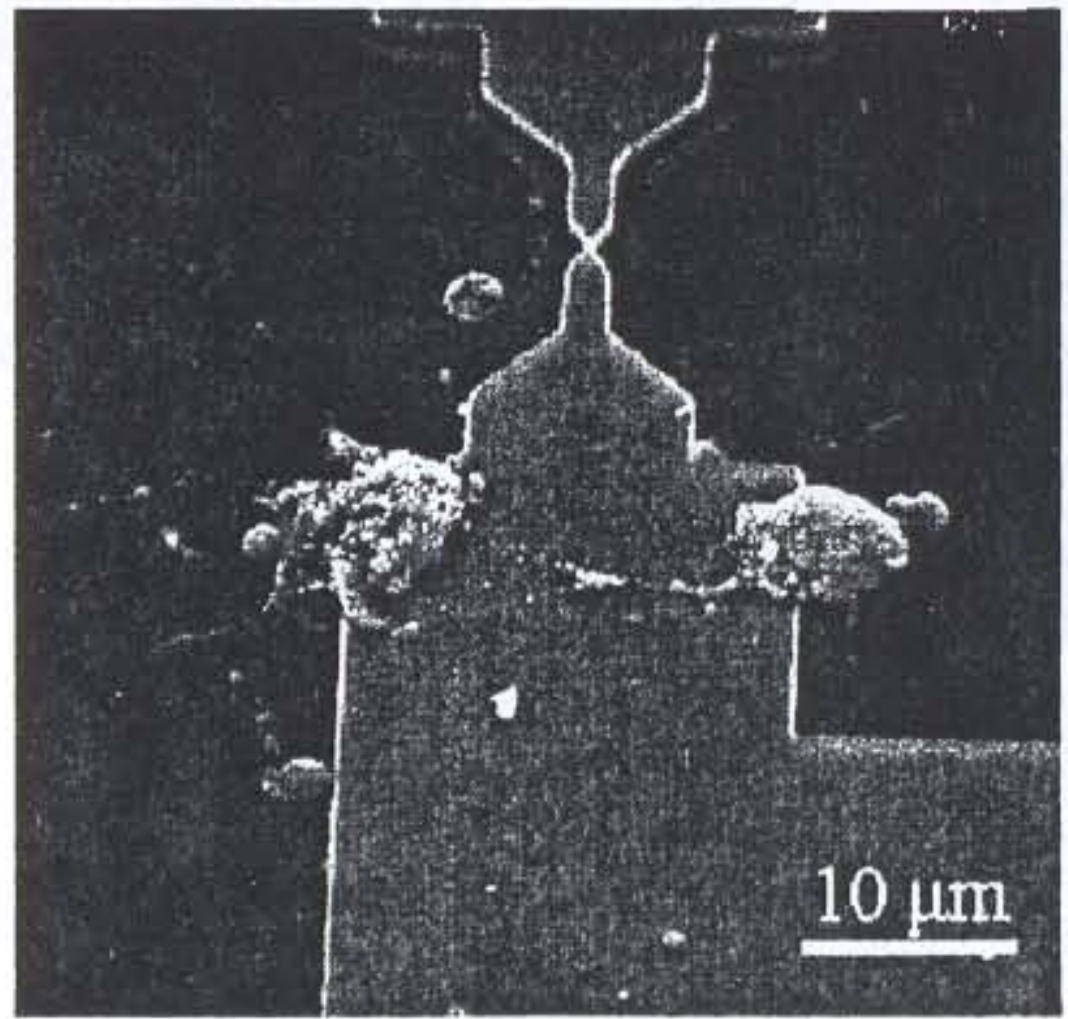

Fig. 5(a) Two neurons on an electrode pad.

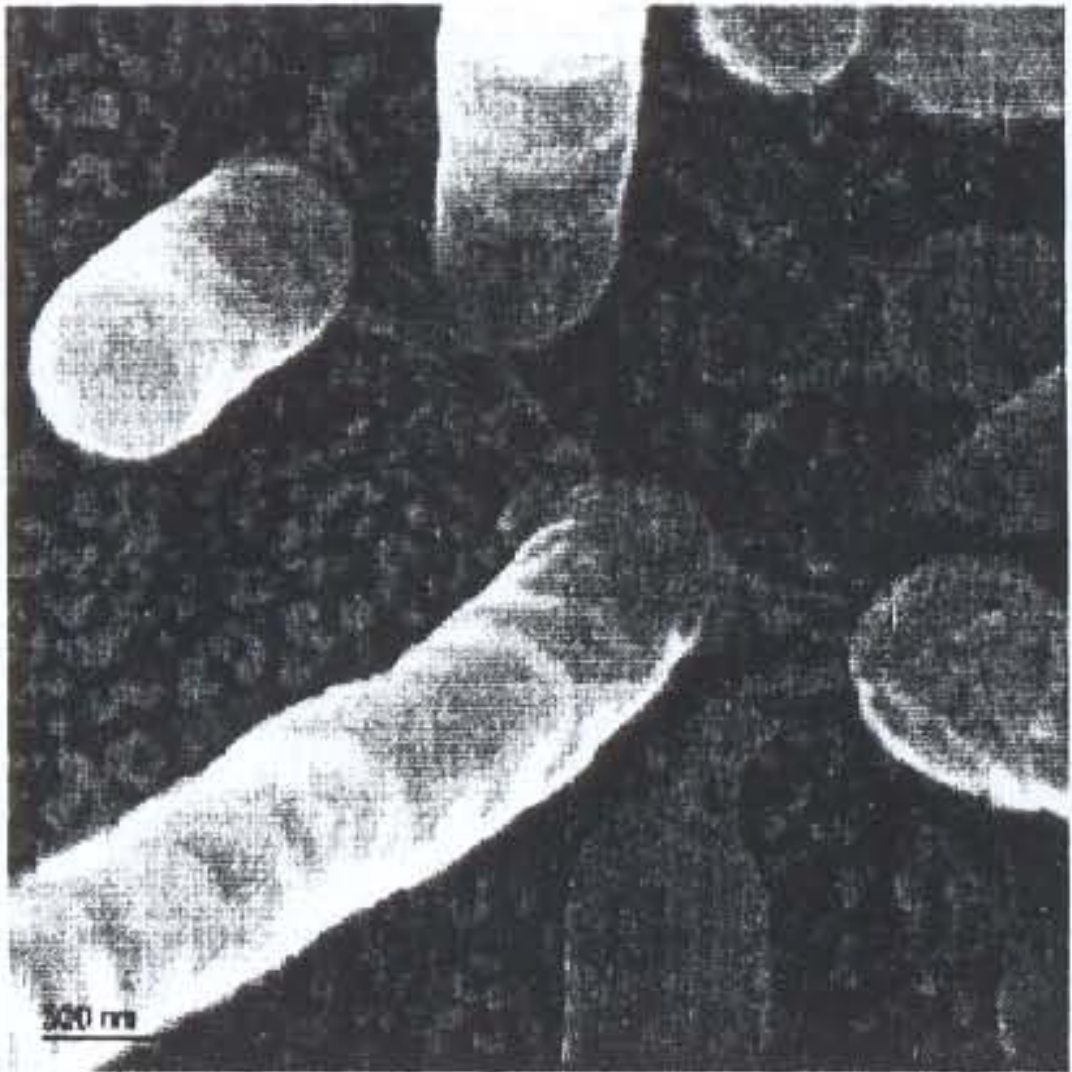

Fig. 4(b) Close-up view of bacteria attachment structures on the nano-pillars.

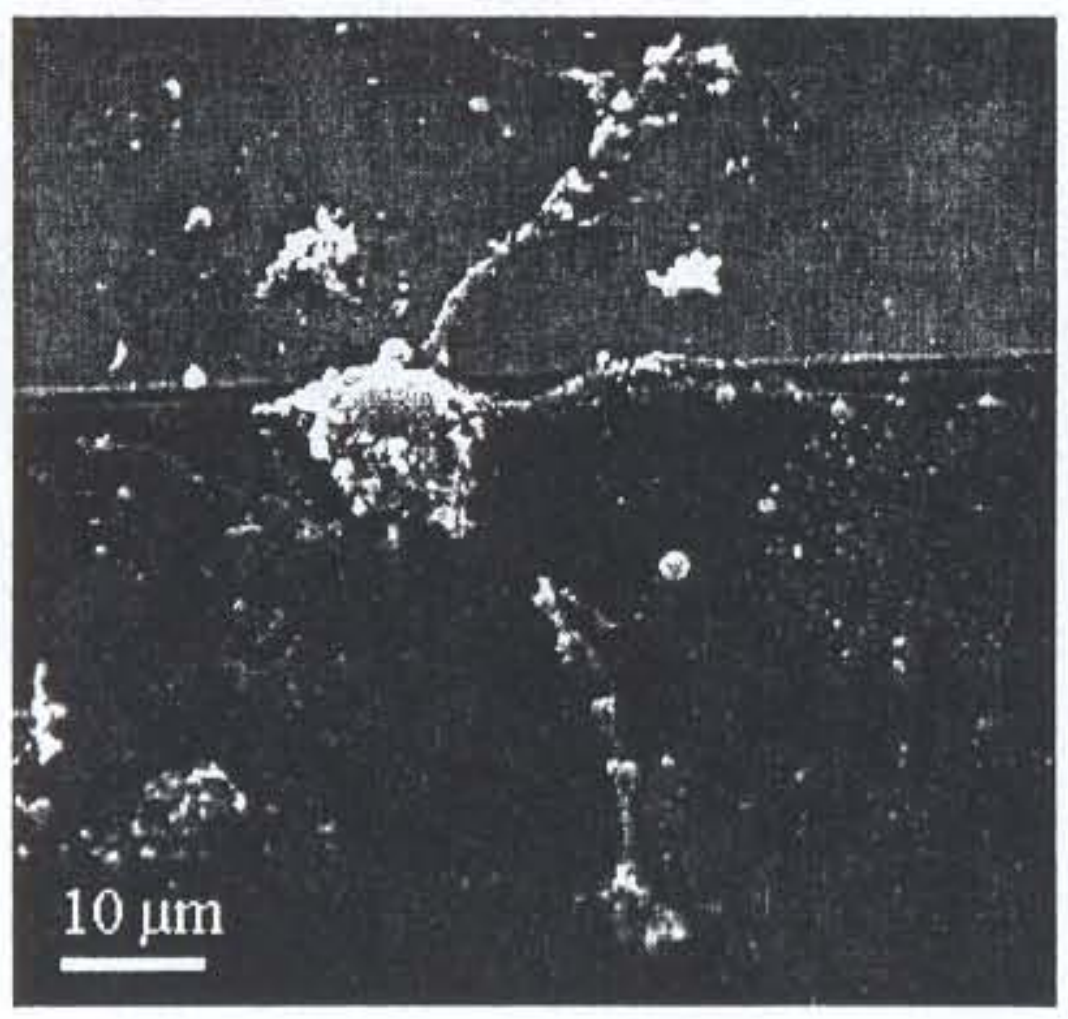

Fig. 5(b) A neuron extending a process onto a nano-pillar region (top).

Neuronal response to electrical stimulation will be recorded from electrodes that have nanostructures and compared to recordings from plain electrodes to study electrode impedance as shown in Fig. 1(b). 


\section{CONCLUSIONS}

High aspect ratio pillar-like polysilicon nanostructures were fabricated. Their diameters ranged from 20 to $100 \mathrm{~nm}$; diameter and packing density are controlled by the ratio of $\mathrm{HBr}$ and $\mathrm{O}_{2}$ in a highly selective silicon RIE. Bacteria preferentially attach to the nano-pillar patterned areas.

In future experiments, neuronal response to electrical stimulation will be recorded from electrodes that have nanostructures and compared to recordings from plain electrodes to study electrode impedance. Applications of these nano-pillars extend beyond electrodes, because these nanostructures may improve integration of tissue with an implanted micro device as cells anchor onto the increased surface area that they provide.

\section{ACKNOWLEDGMENTS}

We would like to thank Hubert Fiumelli and Gordon Vrdoljak for their assistance. This work was supported by a grant from the Whitaker Foundation. All microfabrication was performed at the UC Berkeley Microfabrication Laboratory. Neurons were obtained as approved by the Animal Care and Use Committee, University of California Berkeley.

\section{REFERENCES}

[1] A. Blau, Ch. Ziegler, M. Heyer, F. Endres, G. Schwutzgebel, T. Matties, T. Stieglitz, J. U. Meyer, and W. Goepel, "Characterization and optimization of microelectrode arrays for in vivo nerve signal recording and stimulation,"iosensors \& Bioelectronics, 12(910):883:892, 1997.

[2] D. A. Stenger and T. M. McKenna, editors. Enabling Technologies for Cultured Neural Networks. Academic Press, New York, 1994.

[3] P. Thiebaud. Fabrication of microelectrode arrays for electrophysiological monitoring of hippocampal organotypic slice cultures by interface. $\mathrm{PhD}$ thesis, Institute of Microtechnology, University of Neuchatel, Switzerland, 1999.

[4] J. D. Weiland and D. J. Anderson, "Chronic Neural Stimulation with Thin-Film, Iridium Oxide Electrodes,"IEEE Transactions on Biomedical Engineering, 47(7): 911-918, July 2000 . 